\title{
Aggregates for Concrete
}

\author{
Dr Peter Taylor, PE
}

National Concrete Pavement

Technology Center

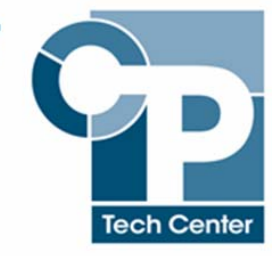

IOWA STATE UNIVERSITY

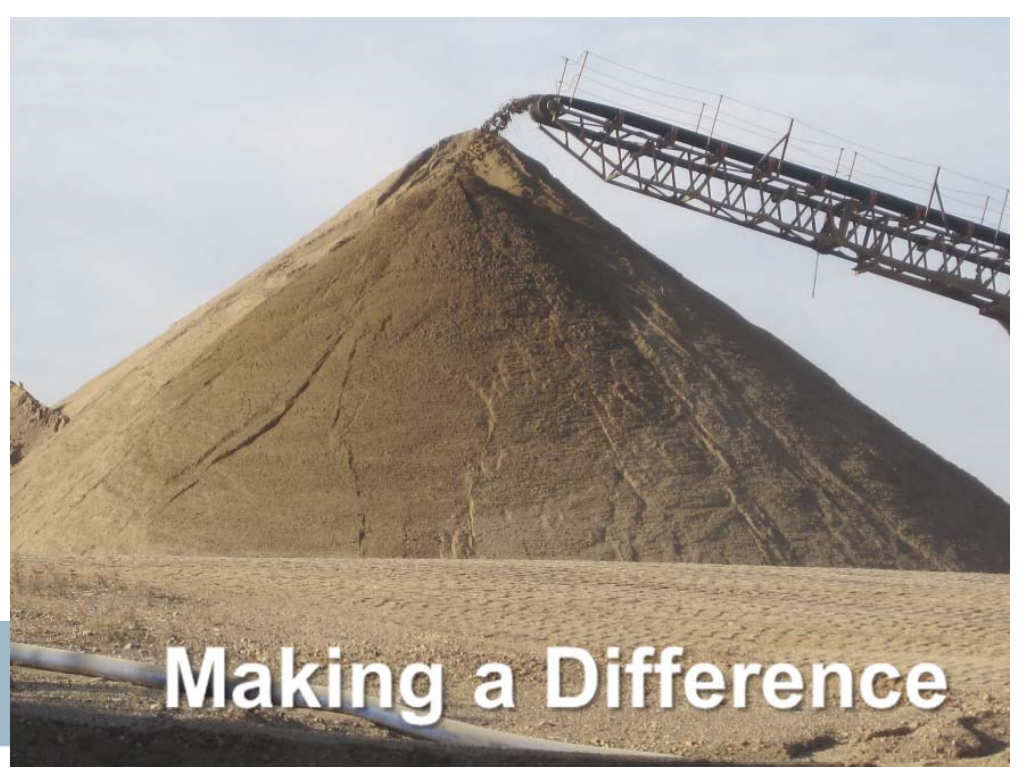




\section{Concrete}

- Civilization would be stunted without it.

- Can be formed to any shape.

- It is fabricated on site.

- Using empirical QC tools.

- There is a lot of it

- Its more complicated than you think

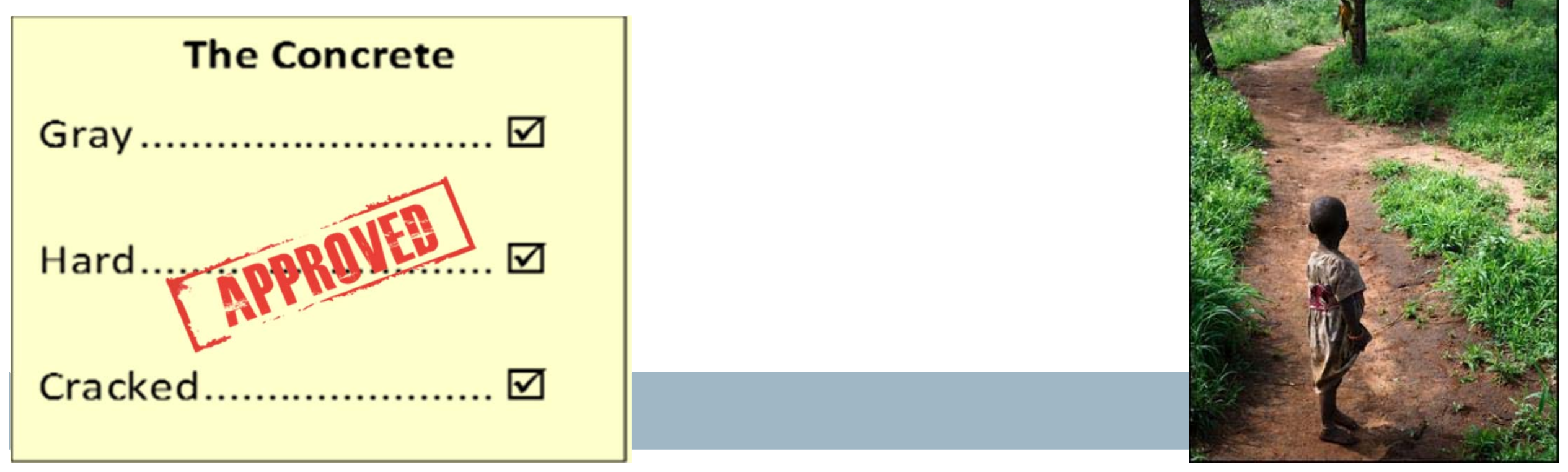




\section{What is Concrete?}
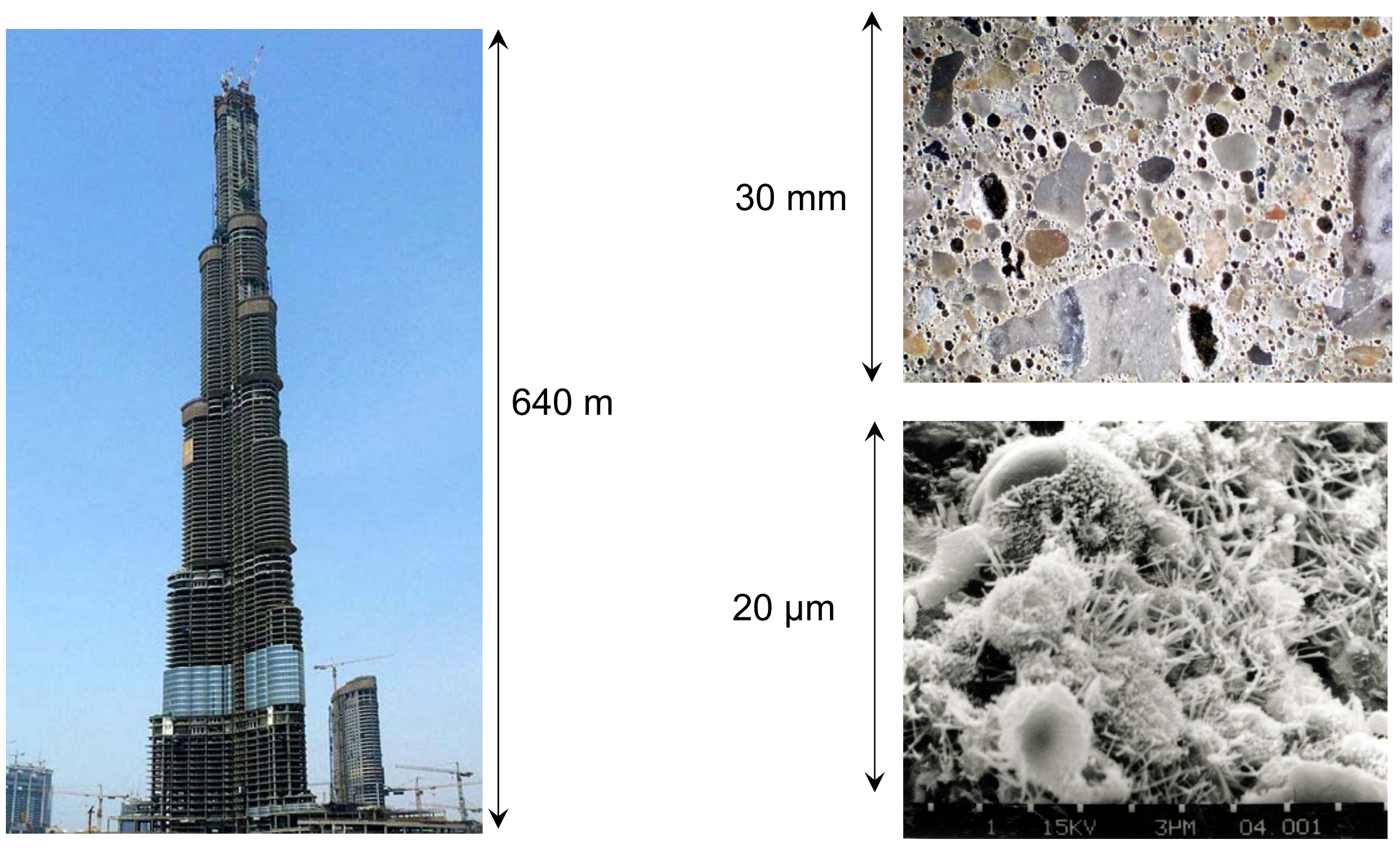


\section{The Perfect Material for Pavements}

- Cost effective

- Easy to build with

- Get traffic on it fast

- Unbreakable

- Weather-proof

- Sustainable

- Resilient

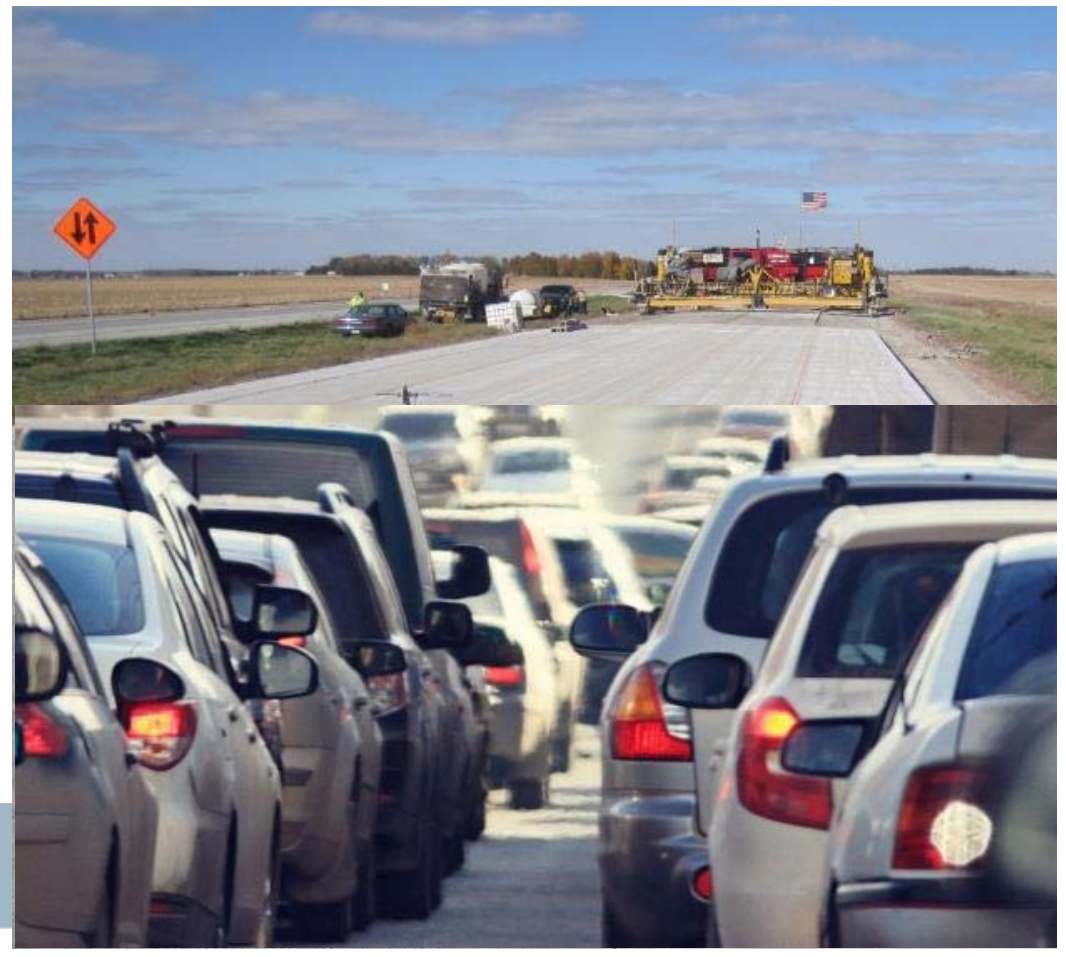




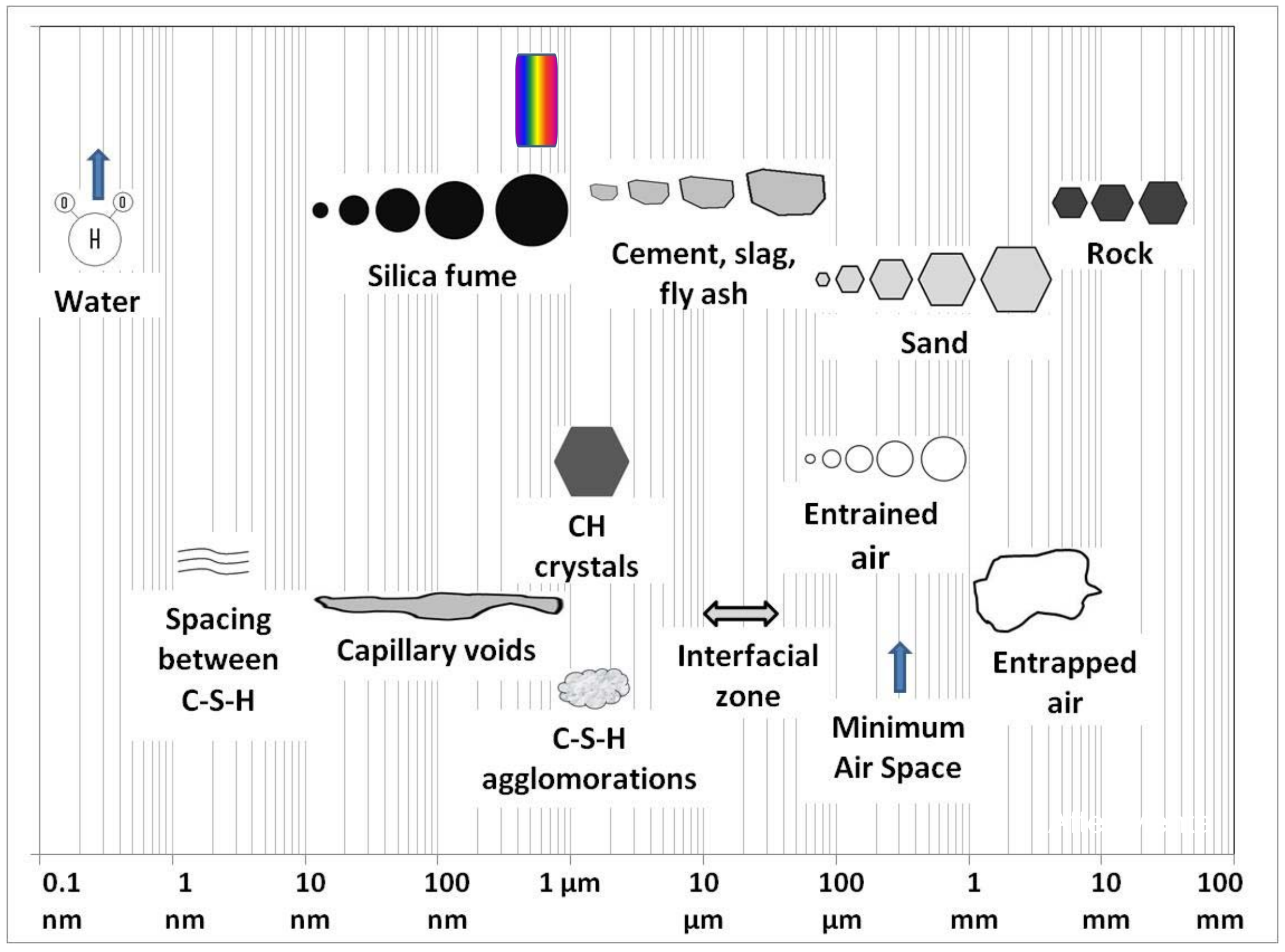




\section{Life is changing}

\begin{tabular}{|c|c|c|}
\hline & 1977 & 2017 \\
\hline No. of ingredients & $\begin{array}{l}\text { Cement, water, } \\
\text { rock, sand, AEA }\end{array}$ & $\begin{array}{l}\text { Add SCMs, } \\
\text { admixtures, int. } \\
\text { aggregates, } \\
\text { limestone... }\end{array}$ \\
\hline Opening & Weeks & Days (or hours) \\
\hline Curing & Weeks & Days \\
\hline De-icing & Sand, $\mathrm{NaCl}$ & $\begin{array}{l}\text { Other chlorides, } \\
\text { formates, acetates }\end{array}$ \\
\hline Design life & 20 years & 100 years \\
\hline Knowledge base & In house & Contracted out \\
\hline
\end{tabular}




\section{Sustainability}

Getting what we need

- Capacity and Longevity

for the minimum :

- Cost

- Energy \& resources

- Pollutants

- Negative impact to society

Simply good engineering (Getting more for less)

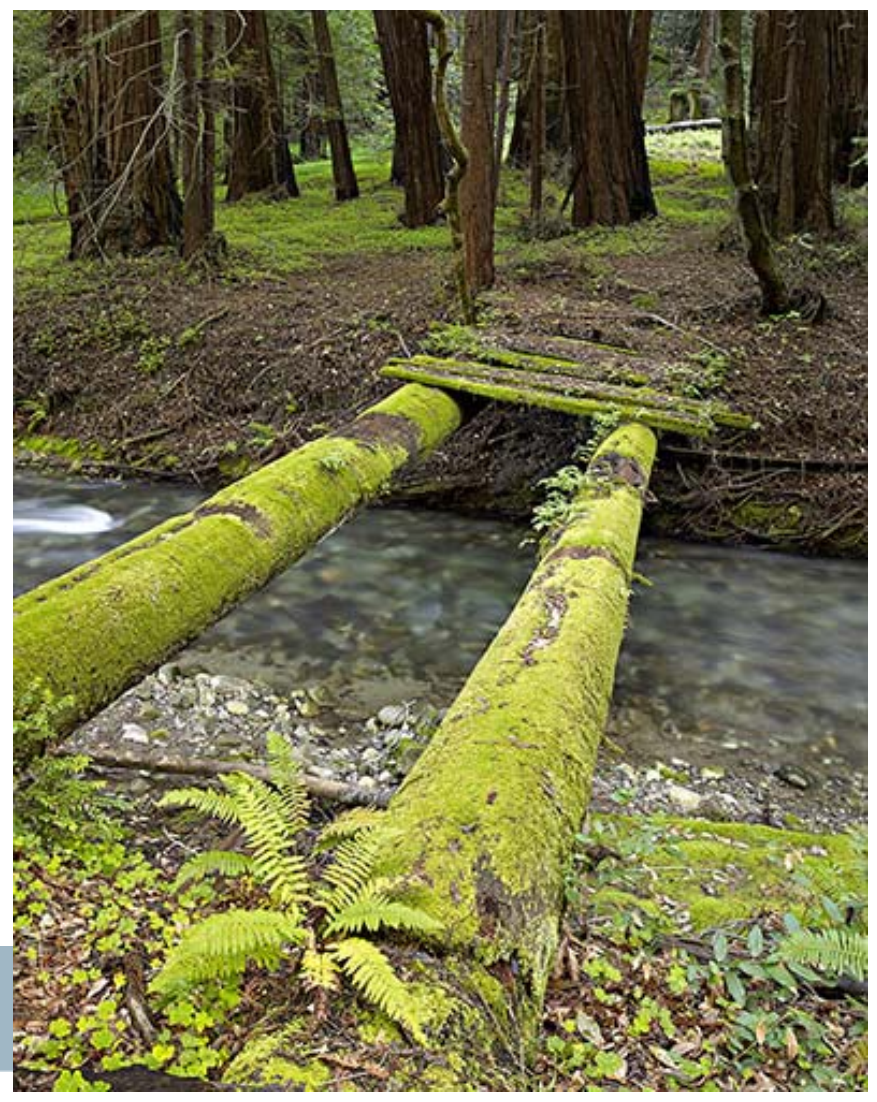




\section{Sustainability}

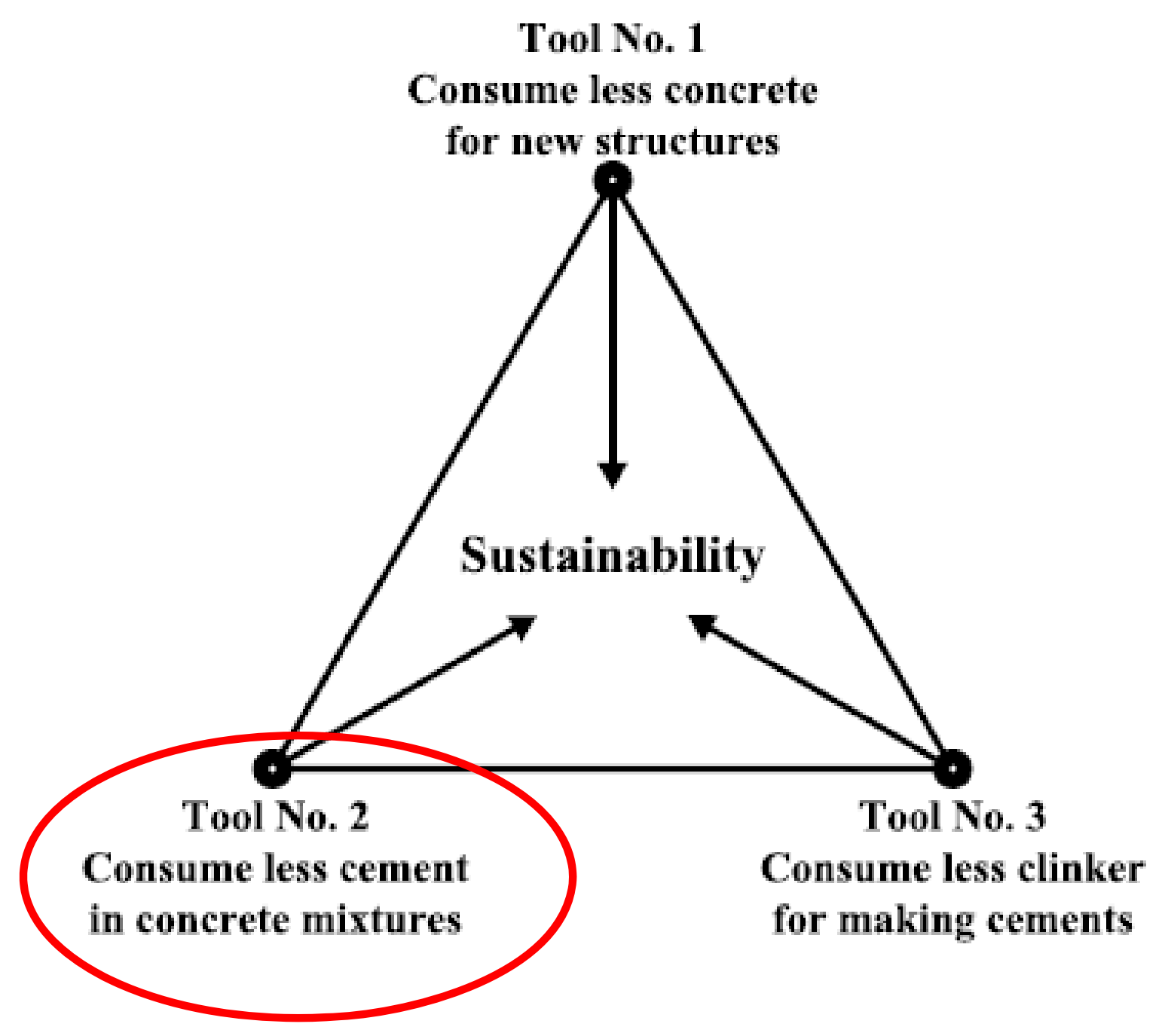




\section{Proportioning}

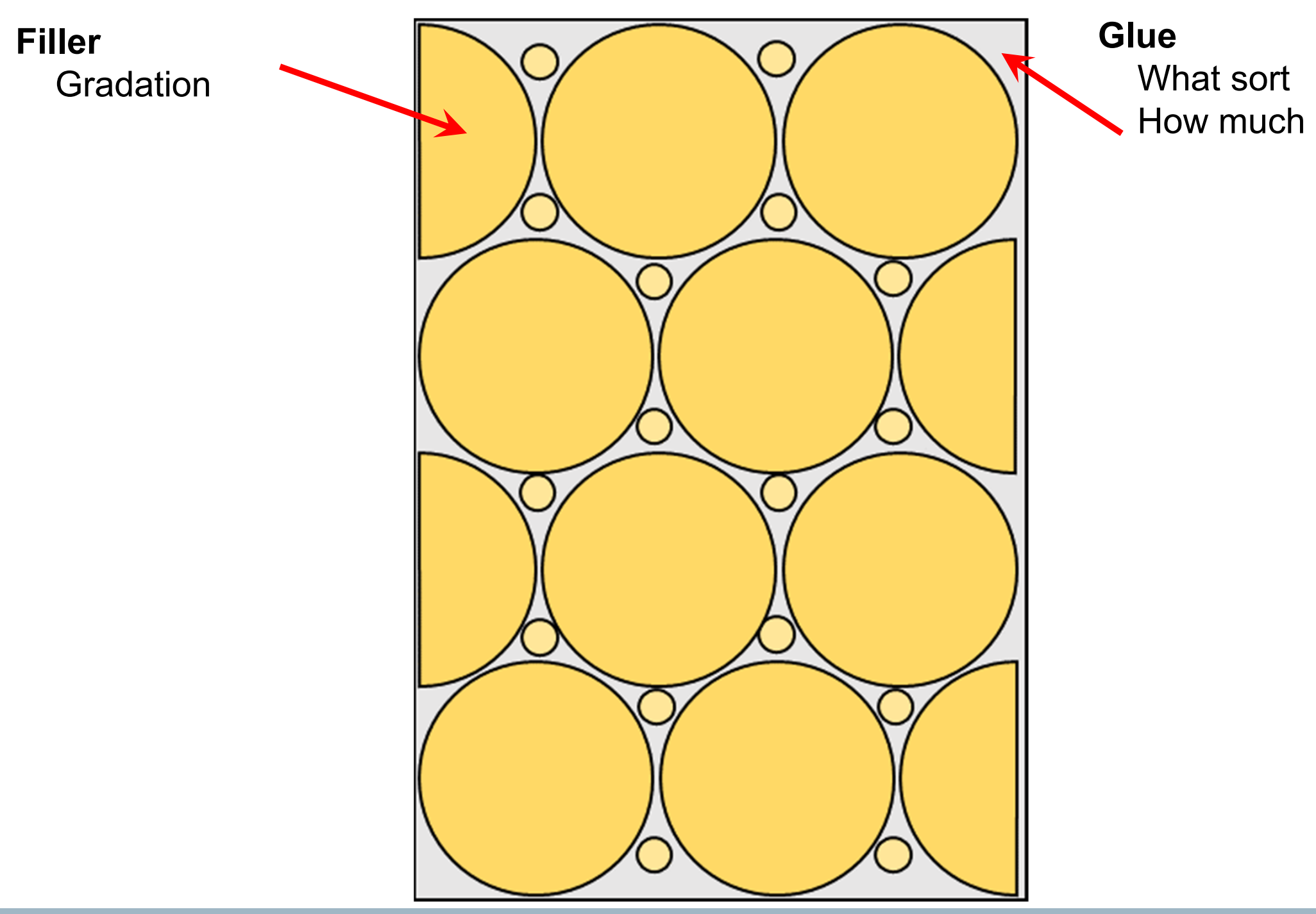




\section{Aggregate Gradation}

\section{- Tarantula Curve}

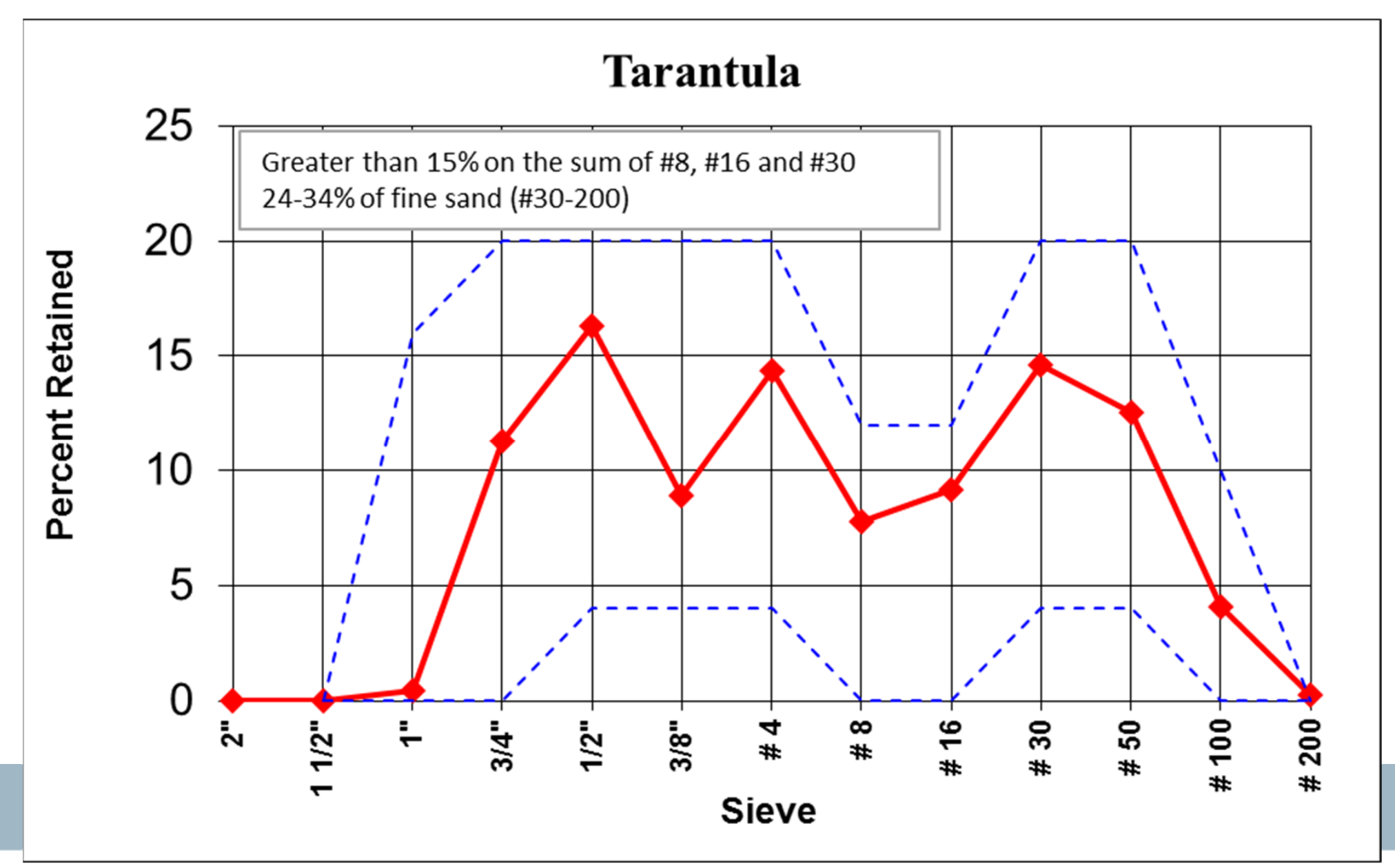




\section{Effect on Binder Content}

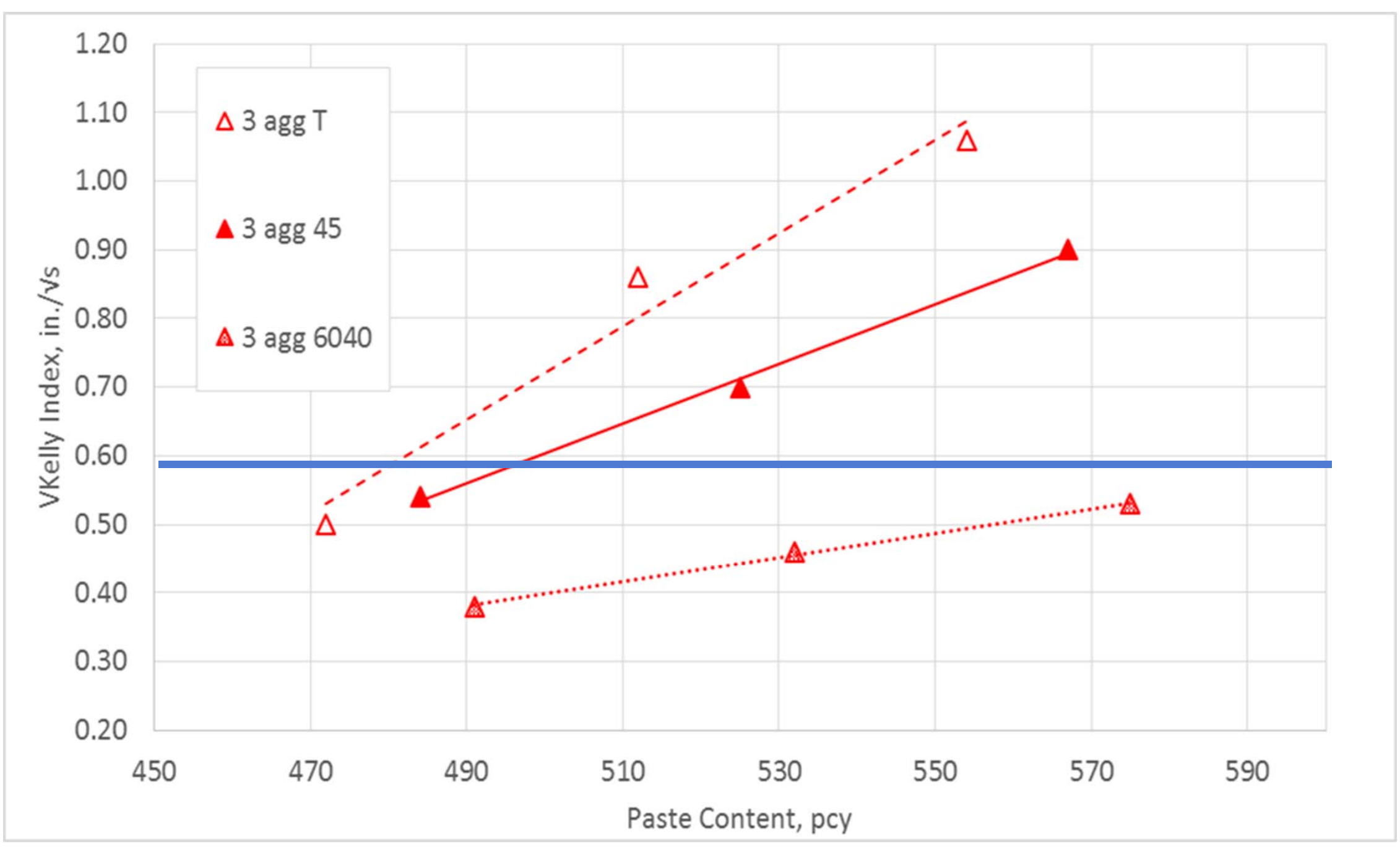




\section{Effect of Proportioning}
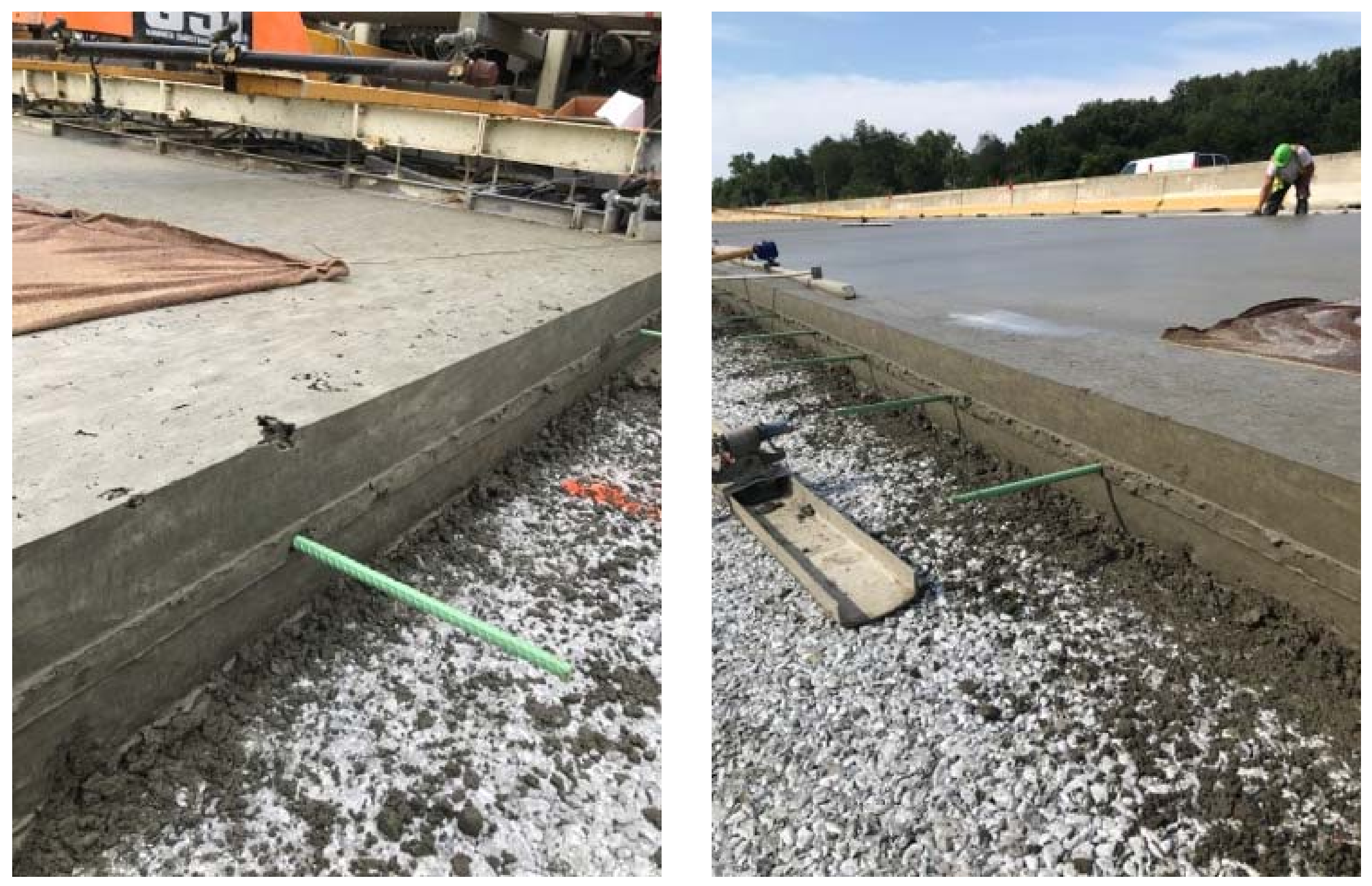


\section{What Can Go Wrong?}

- Alkali Aggregate Reaction

- Reactive aggregates

- Alkali hydroxides

- Water
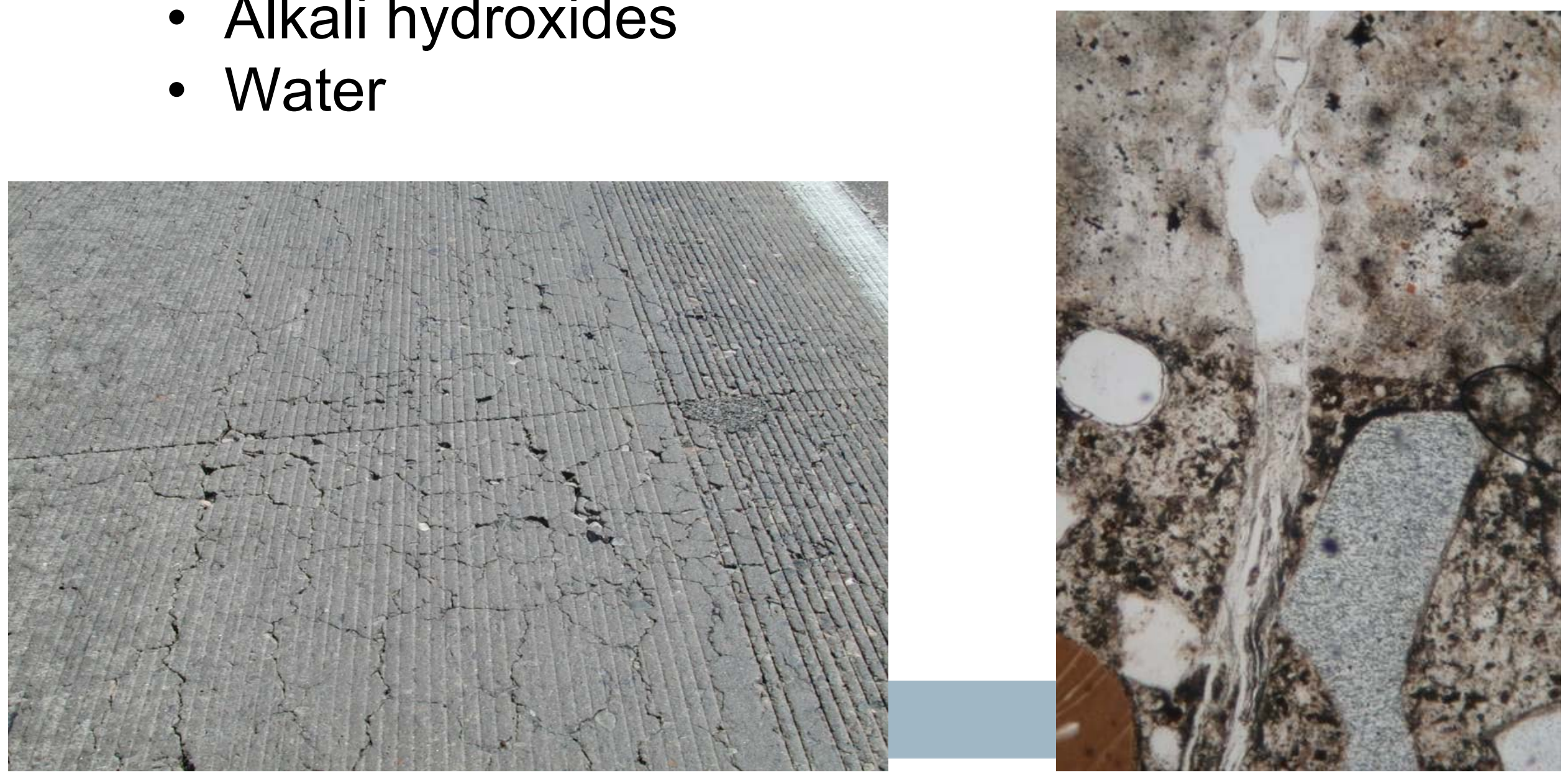


\section{What Can Go Wrong?}

- D-Cracking

- Some limestone aggregates

- Cold weather

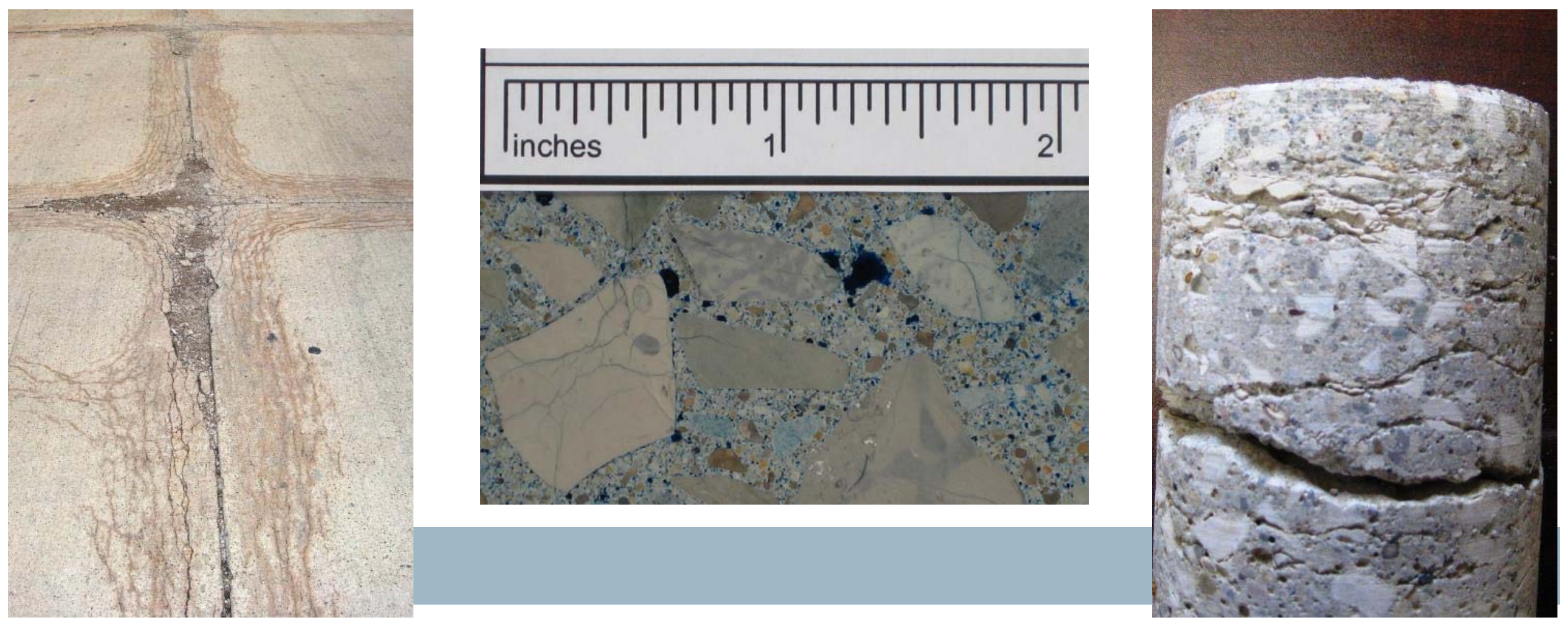




\section{What Can Go Wrong?}

- Alkali Carbonate Reaction - A mystery

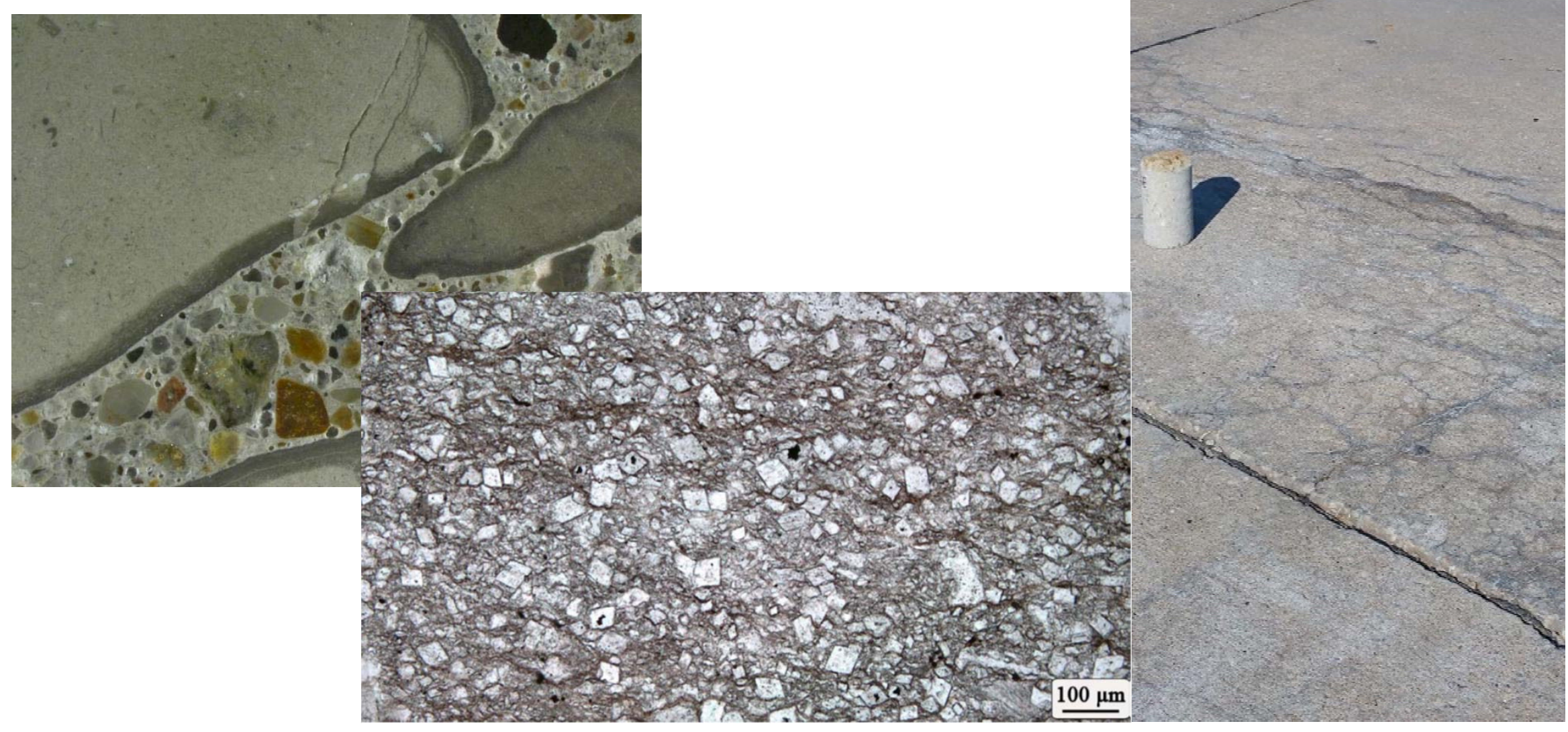




\section{What Can Go Wrong?}

- Popouts

- Porous aggregates

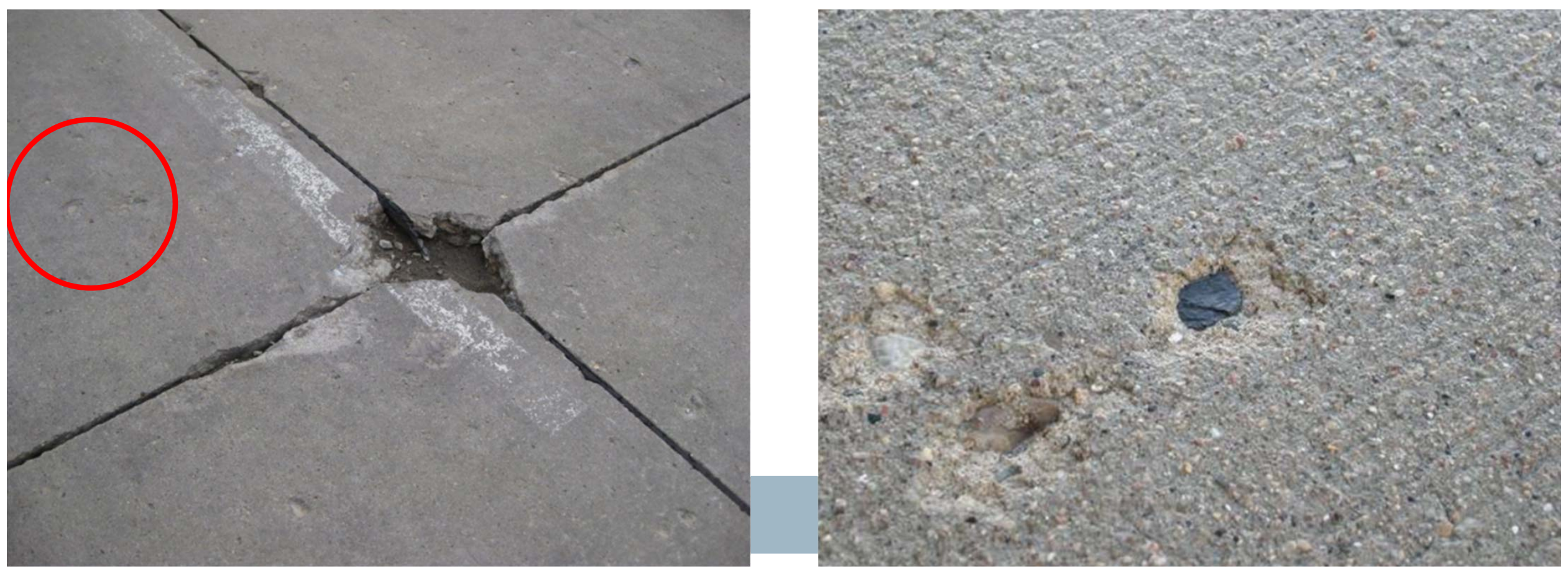




\section{A Better Specification}

- AASHTO PP84 published in March

- Guide Specification

- "Deemed to satisfy"

- Avoids bonus discussion - that is local

- Provisional = meaning we can modify as we learn things

Delivering cancrete ta suruive it's enwiranment 


\section{Require the things that matter}

- Transport properties (everywhere)

- Aggregate stability (everywhere)

- Strength (everywhere)

- Cold weather resistance (cold locations)

- Shrinkage (dry locations)

- Workability (everywhere)

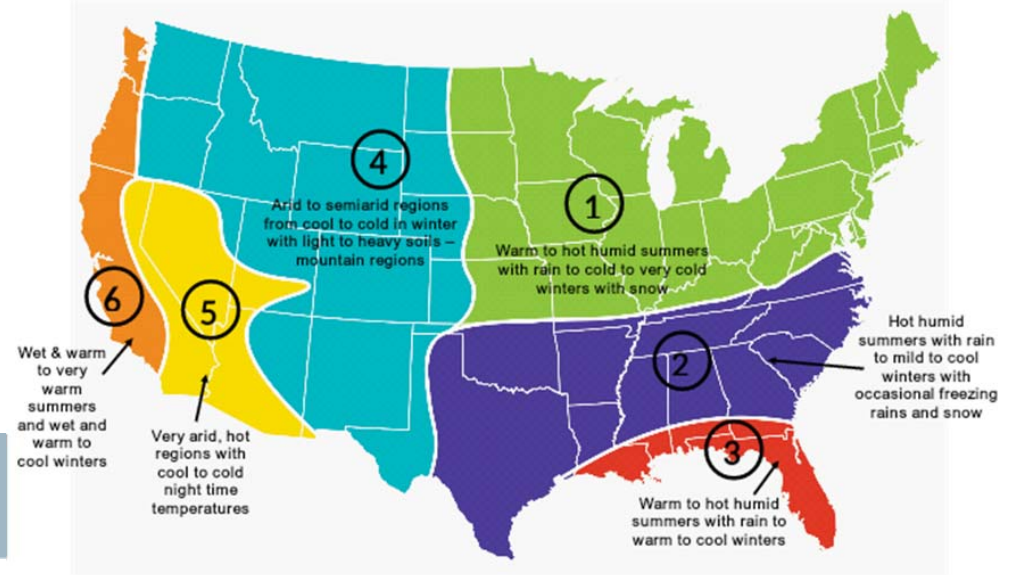




\section{Closing}

- We need to talk...

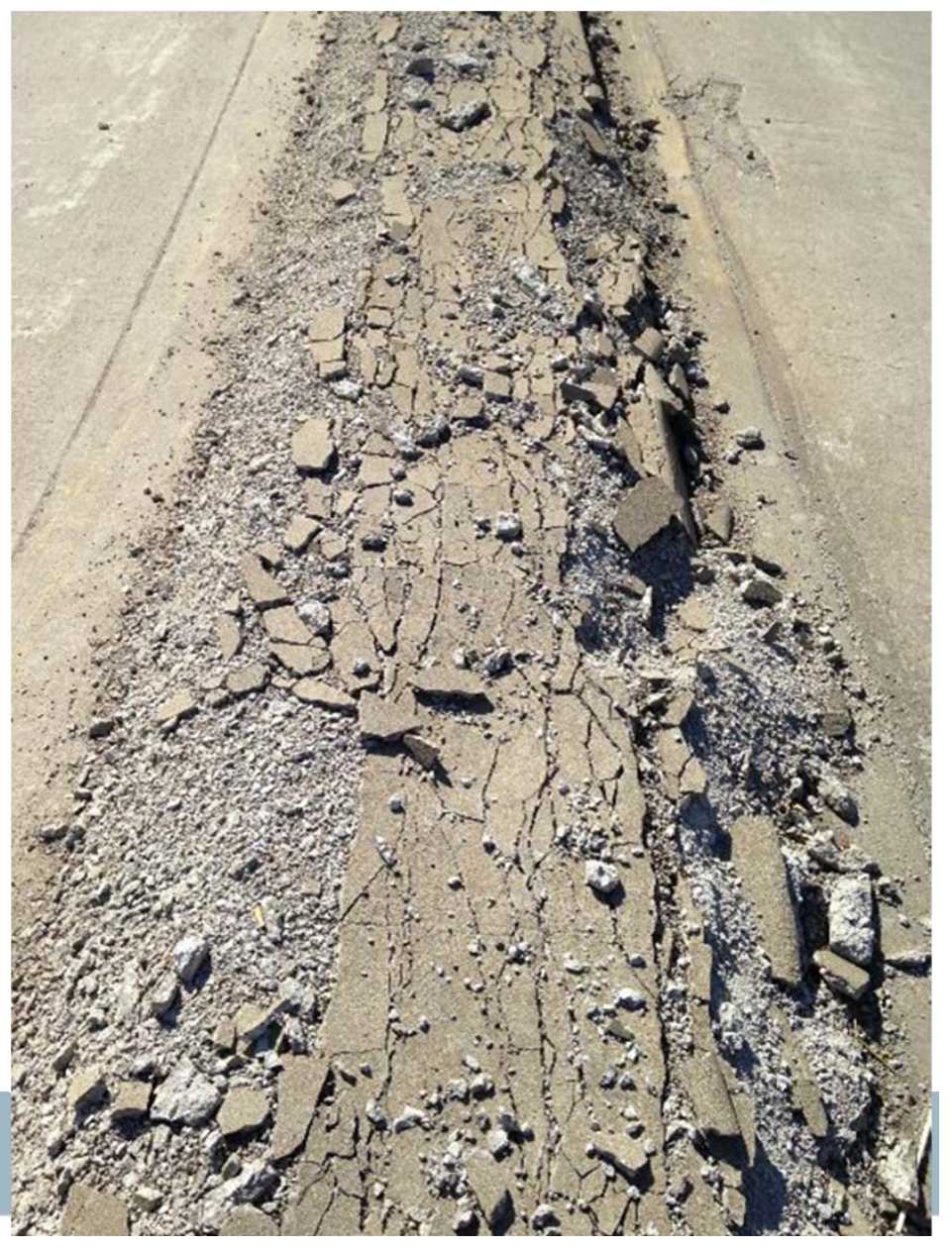

